\title{
Families of Vector Fields which Generate the Group of Diffeomorphisms
}

\author{
A. A. Agrachev* \\ M. Caponigro ${ }^{\dagger}$
}

\begin{abstract}
Given a compact manifold $M$, we prove that any bracket generating and invariant under multiplication on smooth functions family of vector fields on $M$ generates the connected component of unit of the group Diff $M$.
\end{abstract}

Let $M$ be a smooth 11 -dimensional compact manifold, Vec $M$ the space of smooth vector fields on $M$ and $\operatorname{Diff}_{0} M$ the group of isotopic to the identity diffeomorphisms of $M$.

Given $f \in \operatorname{Vec} M$, we denote by $t \mapsto e^{t f}, t \in \mathbb{R}$, the flow on $M$ generated by $f$; then $e^{t f}, t \in \mathbb{R}$, is a one-parametric subgroup of $\operatorname{Diff}_{0} M$. Let $\mathcal{F} \subset$ Vec $M$; the subgroup of $\operatorname{Diff}_{0} M$ generated by $e^{t f}, f \in \mathcal{F}, t \in \mathbb{R}$, is denoted by $\operatorname{Gr} \mathcal{F}$.

Theorem. Let $\mathcal{F} \subset \operatorname{Vec} M$; if $\mathrm{Gr} \mathcal{F}$ acts transitively on $M$, then

$$
\operatorname{Gr}\left\{a f: a \in C^{\infty}(M), f \in \mathcal{F}\right\}=\operatorname{Diff}_{0} M
$$

Corollary 1. Let $\Delta \subset T M$ be a completely nonholonomic vector distribution. Then any isotopic to the identity diffeomorphism of $M$ has a form $e^{f_{1}} \circ \cdots \circ e^{f_{k}}$, where $f_{1}, \ldots, f_{k}$ are sections of $\Delta$.

Remark. Recall that $\operatorname{Gr}\left\{f_{1}, f_{2}\right\}$ acts transitively on $M$ for a generic pair of smooth vector fields $f_{1}, f_{2}$.

\footnotetext{
${ }^{*}$ SISSA, Trieste \& Steklov Math. Inst., Moscow

${ }^{\dagger}$ SISSA, Trieste

${ }^{1}$ In this paper, smooth means $C^{\infty}$.
} 
We start the proof of the theorem with an auxiliary lemma that is actually the main part of the proof. Let $B \subset \mathbb{R}^{n}$ be diffeomorphic to a cube, $0 \in B$; we set $C_{0}^{\infty}(B)=\left\{a \in C^{\infty}(B): a(0)=0\right\}$ and assume that $C_{0}^{\infty}(B)$ is endowed with the standard $C^{\infty}$-topology.

Lemma 1 (Main Lemma). Let $X_{i} \in \operatorname{Vec}^{n}, a_{i} \in C^{\infty}\left(\mathbb{R}^{n}\right), i=1, \ldots, n$, and the following conditions hold:

- $\operatorname{span}\left\{X_{1}(0), \ldots, X_{n}(0)\right\}=\mathbb{R}^{n}$,

- $a_{i}(0)=0,\left\langle d_{0} a_{i}, X_{i}(0)\right\rangle<0, i=1, \ldots, n$;

then there exist $\epsilon, \varepsilon>0$ and a neighborhood $\mathcal{O}$ of $\left.\left(\epsilon a_{1}, \ldots, \epsilon a_{n}\right)\right|_{B_{\varepsilon}}$ in $C_{0}^{\infty}\left(B_{\varepsilon}\right)^{n}$ such that the mapping

$$
\Phi:\left.\left(b_{1}, \ldots, b_{n}\right) \mapsto\left(e^{b_{1} X_{1}} \circ \cdots \circ e^{b_{n} X_{n}}\right)\right|_{B_{\varepsilon}}
$$

is an open map from $\mathcal{O}$ into $C_{0}^{\infty}\left(B_{\varepsilon}\right)^{n}$, where

$$
B_{\varepsilon}=\left\{e^{s_{1} X_{1}} \circ \cdots \circ e^{s_{n} X_{n}}(0):\left|s_{i}\right| \leq \varepsilon, i=1, \ldots, n\right\} .
$$

Sketch of proof. Openness of the map (1) is derived from the Hamilton's version of the Nash-Moser inverse function theorem [2]. Set $\bar{a}=$ $\left(\epsilon a_{1}, \ldots, \epsilon a_{n}\right)$. In order to apply the Nash-Moser theorem we have to invert the differential of $\Phi$ at $\bar{a}$ and show that inverse is "tame" with respect to $\bar{a}$. Here we make computations only for fixed $\bar{a}$ and leave the boring check of the tame dependence on $\bar{a}$ for the detailed paper.

Note that $e^{\epsilon a_{j} X_{j}}$ are closed to identity diffeomorphisms, hence $\left.\frac{\partial \Phi}{\partial b_{i}}\right|_{\bar{a}}$ is obtained from $\left.\frac{\partial}{\partial b_{i}} e^{b_{i} X_{i}}\right|_{\epsilon a_{i}}$ by a closed to identity change of variables. We have

$$
\left(\frac{\partial}{\partial a} e^{a X}\right): b \mapsto e_{*}^{a X}\left(\int_{0}^{1} e^{\int_{t}^{0}\langle d a, X\rangle \circ e^{\tau a X} d \tau} b \circ e^{t a X} d t X\right) \circ e^{a X}
$$

This equality follows from the standard "variations formula" (see [1]) and the relation:

$$
\left(e^{t a X}\right)_{*}: X \mapsto\left(e^{\int_{0}^{t}\langle d a, X\rangle \circ e^{-\tau a X} d \tau}\right) X
$$

Let us define an operator $A(a, X): C_{0}^{\infty}\left(\hat{B}_{\varepsilon}\right) \rightarrow C_{0}^{\infty}\left(\hat{B}_{\varepsilon}\right)$ by the formula

$$
A(a, X) b=\int_{0}^{1} e^{\int_{t}^{0}\langle d a, X\rangle \circ e^{\tau a X} d \tau} b \circ e^{t a X} d t
$$


where $\hat{B}_{\varepsilon}=\left\{e^{s X}(x):|s| \leq \varepsilon, x \in \Pi^{n-1}\right\}$ and $\Pi^{n-1}$ is a transversal to $X$ small $(n-1)$-dimensional box. We see that invertibility of $A\left(\varepsilon a_{i}, X_{i}\right), i=$ $1, \ldots, n$, implies invertibility of $D_{\bar{a}} \Phi$.

Now set $\mathcal{X}=\left\{b X: b \in C^{\infty}(M)\right\} \subset$ Vec $M$. The map

$$
(b X) \mapsto(A(a, x) b) X
$$

has a clear intrinsic meaning as a linear operator on the space $\mathcal{X}$; moreover, this operator depends only on the vector field $a X \in \mathcal{X}$. Indeed,

$$
(A(a, X) b) X=e_{*}^{-a X}\left(\left.D_{(a X)} \operatorname{Exp}\right|_{\mathcal{X}}(b X)\right) \circ e^{-a X},
$$

where $D_{Y} \operatorname{Exp}$ is the differential at the point $Y \in \operatorname{Vec} M$ of the map

$$
\operatorname{Exp}: Y \mapsto e^{Y}, \quad Y \in \operatorname{Vec} M .
$$

Recall that $a(0)=0,\left\langle d_{0} a, X(0)\right\rangle<0$. In particular, $X$ is transversal to the hypersurface $a^{-1}(0)$. We may rectify the field $X$ in such a way that, in new coordinates, $X=\frac{\partial}{\partial x_{1}}, a\left(0, x_{2}, \ldots, x_{n}\right)=0$. Now the field $a X$ can be treated as a depending on $y=\left(x_{2}, \ldots, x_{n}\right)$ family of 1-dimensional vector fields $a\left(x_{1}, y\right) \frac{\partial}{\partial x_{1}}$. Moreover, $a(0, y)=0, \quad \frac{\partial a}{\partial x_{1}}(0, y)=\alpha(y)<0$.

A hyperbolic 1-dimensional field $a\left(x_{1}, y\right) \frac{\partial}{\partial x_{1}}$ can be linearized by a smooth change of variable and this smooth change of variable smoothly depends on $y$. Hence we may assume that $a X=\alpha(y) x_{1} \frac{\partial}{\partial x_{1}}$. Then $b \circ e^{t a X}\left(x_{1}, y\right)=$ $b\left(e^{\alpha(y) t} x_{1}, y\right)$.

We thus have to invert the operator

$$
\hat{A}: b\left(x_{1}, y\right) \mapsto \int_{0}^{1} e^{-t \alpha(y)} b\left(e^{\alpha(y) t} x_{1}, y\right) d t
$$

acting in the space of smooth functions on a box. We can write

$$
b\left(x_{1}, y\right)=b_{0}(y)+x_{1} b_{1}(y)+x_{1}^{2} u\left(x_{1}, y\right),
$$

where $u$ is a smooth function. Then $\hat{A} b_{0}=\frac{1}{\alpha}\left(1-e^{-\alpha}\right) b_{0}, \hat{A}\left(x_{1} b_{1}\right)=x_{1} b_{1}$ and

$$
\hat{A}\left(x_{1}^{2} u\left(x_{1}, y\right)\right)=x_{1}^{2} \int_{0}^{1} e^{\alpha(y) t} u\left(e^{\alpha(y) t} x_{1}, y\right) d t=-\frac{x_{1}^{2}}{\alpha(y)} \int_{e^{\alpha(y)}}^{1} u\left(\tau x_{1}, y\right) d \tau .
$$


What remains is to invert the operator

$$
B: u\left(x_{1}, y\right) \mapsto \int_{e^{\alpha(y)}}^{1} u\left(\tau x_{1}, y\right) d \tau .
$$

We set $v\left(x_{1}, y\right)=\frac{1}{x_{1}} \int_{0}^{x_{1}} u(s, y) d s$; then

$$
(B u)\left(x_{1}, y\right)=\left(v\left(x_{1}, y\right)-e^{\alpha(y)} v\left(e^{\alpha(y)} x_{1}, y\right)\right) .
$$

We introduce one more operator:

$$
R: v\left(x_{1}, y\right) \mapsto e^{\alpha(y)} v\left(e^{\alpha(y)} x_{1}, y\right) .
$$

Let $\|v\|_{C^{k, 0}}=\sup _{1 \leq i \leq k}\left\|\frac{\partial^{i} v}{\partial x_{1}^{i}}\right\|_{C^{0}}$. Obviously, $\|R\|_{C^{k, 0}} \leq e^{\sup \alpha}<1, \forall k$. Hence $(I-R)^{-1}$ transforms a smooth on the box function $\psi$ in the function $\varphi=$ $(I-R)^{-1} \psi$ that is smooth with respect to $x_{1}$. As usually, the chain rule for the differentiation allows to demonstrate that function $\varphi$ is also smooth on the box and to compute its derivatives:

$$
\frac{\partial \varphi}{\partial y_{i}}=(I-R)^{-1}\left(\frac{\partial \psi}{\partial y_{i}}-e^{\alpha} \frac{\partial \alpha}{\partial y_{i}} \varphi-e^{2 \alpha} \frac{\partial \alpha}{\partial y_{i}} \frac{\partial \varphi}{\partial x_{1}}\right) \text {, e.t.c. }
$$

Coming back to equation (2), we obtain: $v=(I-R)^{-1} B u$. Finally,

$$
B^{-1}: w \mapsto \frac{\partial}{\partial x_{1}}\left(x_{1}(I-R)^{-1} w\right)
$$

Now set

$$
\mathcal{P}=\operatorname{Gr}\left\{a f: a \in C^{\infty}(M), f \in \mathcal{F}\right\}, \quad \mathcal{P}_{q}=\{P \in \mathcal{P}: P(q)=q\}, q \in M .
$$

Lemma 2. Any $q \in M$ possesses a neighborhood $U_{q} \subset M$ such that the set

$$
\left\{\left.P\right|_{U_{q}}: P \in \mathcal{P}_{q}\right\}
$$

has a nonempty interior in $C_{q}^{\infty}\left(U_{q}, M\right)$, where $C_{q}^{\infty}\left(U_{q}, M\right)$ is the Fréchet manifold of smooth maps $F: U_{q} \rightarrow M$ such that $F(q)=q$. 
Proof. According to the Orbit Theorem of Sussmann [4] (see also the textbook [1]), transitivity of the action of $\mathrm{Gr} \mathcal{F}$ on $M$ implies that

$$
T_{q} M=\operatorname{span}\left\{P_{*} f(q): p \in \operatorname{Gr} \mathcal{F}, f \in \mathcal{F}\right\} .
$$

Take $X_{i}=P_{i *} f_{i}, i=1, \ldots, n$, such that $P_{i} \in \operatorname{Gr} \mathcal{F}, f_{i} \in \mathcal{F}$, and $X_{1}(q), \ldots, X_{n}(q)$ form a basis of $T_{q} M$. Then for any vanished at $q$ smooth functions $a_{1}, \ldots, a_{n}$, the diffeomorphism

$$
e^{a_{1} X_{1}} \circ \cdots \circ e^{a_{n} X_{n}}=P_{1} \circ e^{\left(a_{1} \circ P_{1}\right) f_{1}} \circ P_{1}^{-1} \circ \cdots \circ P_{n} \circ e^{\left(a_{n} \circ P_{n}\right) f_{n}} \circ P_{n}^{-1}
$$

belongs to the group $\mathcal{P}_{q}$. The desired result now follows from Main Lemma.

Corollary 2. Interior of the set (3) contains the identical map.

Proof. Let $\mathcal{O}$ be an open subset of $C_{q}^{\infty}\left(U_{q}, M\right)$ that is contained in (3) and $\left.P_{0}\right|_{U_{q}} \in \mathcal{O}$. Then $P_{0}^{-1} \circ \mathcal{O}$ is a contained in (3) neighborhood of the identity.

Definition 1. Given $P \in \operatorname{Diff} M$, we set supp $P=\overline{\{x \in M: P(x) \neq x\}}$.

Lemma 3. Let $\mathcal{O}$ be a neighborhood of the identity in $\operatorname{Diff} M$. Then for any $q \in M$ and any neighborhood $U_{q} \subset M$ of $q$, we have:

$$
q \in \operatorname{int}\left\{P(q): P \in \mathcal{O} \cap \mathcal{P}, \text { supp } P \subset U_{q}\right\} .
$$

Proof. Let vector fields $X_{1}, \ldots, X_{n}$ be as in the proof of Lemma 2 and $b \in C^{\infty}(M)$ a cut-off function such that supp $b \subset U_{q}$ and $q \in$ int $^{-1}(1)$. Then the diffeomorphism

$$
Q\left(s_{1}, \ldots, s_{n}\right)=e^{s_{1} b X_{1}} \circ \cdots \circ e^{s_{n} b X_{n}}
$$

belongs to $\mathcal{O} \cap \mathcal{P}$ for all sufficiently close to 0 real numbers $s_{1}, \ldots, s_{n}$ and $\operatorname{supp} Q\left(s_{1}, \ldots, s_{n}\right) \subset U_{q}$. On the other hand, the map

$$
\left(s_{1}, \ldots, s_{n}\right) \mapsto Q\left(s_{1}, \ldots, s_{n}\right)(q)
$$

is a local diffeomorphism in a neighborhood of 0 .

Lemma 4. Let $\bigcup_{j} U_{j}=M$ be a covering of $M$ by open subsets and $\mathcal{O}$ be a neighborhood of identity in $\operatorname{Diff} M$. Then the group $\operatorname{Diff}_{0} M$ is generated by the subset

$$
\left\{P \in \mathcal{O}: \exists j \text { such that supp } P \subset U_{j}\right\} .
$$


Proof. The group $\operatorname{Diff}_{0} M$ is obviously generated by any neighborhood of the identity. We may assume that the covering of $M$ is finite and any $U_{j}$ is contained in a coordinate neighborhood. Moreover, taking a finer covering and a smaller neighborhood $\mathcal{O}$ if necessary, we may assume that for any $P \in \mathcal{O}$ and any $U_{j}$, the coordinate representation of $\left.P\right|_{U_{j}}$ has a form $P: x \mapsto$ $x+\varphi_{P}(x)$, where $\varphi$ is a $C^{1}$-small smooth vector function.

Now consider a refined covering $\bigcup_{i} O_{i}=M$, so that $\bar{O}_{i} \subset U_{j_{i}}$ for some $j_{i}$ and cut-off functions $a_{i}$ such that $\left.a_{i}\right|_{O_{i}}=1$, supp $a_{i} \subset U_{j_{i}}$. Given $P \in \mathcal{O}$, we set

$$
P_{i}(x)=x+a_{i}(x) \varphi_{P}(x), \forall x \in U_{j_{i}} \text { and } P_{i}(q)=q, \forall q \in M \backslash U_{j_{i}} .
$$

Then supp $\left(P_{i}^{-1} \circ P\right) \subset \operatorname{supp} P \backslash O_{i}$. Now, by the induction with respect to $i$, we step by step arrive to a diffeomorphism with empty support. In other words, we present $P$ as a composition of diffeomorphisms whose supports are contained in $U_{j}$.

Proof of the Theorem. According to Lemma 4, it is sufficient to prove that there exist a neighborhood $U_{q} \subset M$ and a neighborhood of the identity $\mathcal{O} \subset \operatorname{Diff} M$ such that any diffeomorphism $P \in \mathcal{O}$ whose support is contained in $U_{q}$ belongs to $\mathcal{P}$. Moreover, Lemma 3 allows to assume that $P(q)=q$. Finally, the corollary to Lemma 2 completes the job.

Acknowledgment. First coauthor is greatful to Boris Khesin who asked him the question answered by this paper (see also recent preprint [3]).

\section{References}

[1] A. A. Agrachev,Yu. L. Sachkov, Control theory from the geometric viewpoint. Springer-Verlag, Berlin, 2004, xiv+412pp.

[2] R. Hamilton, The inverse function theorem of Nash and Moser. Bulletin of the Amer. Math. Soc., 1982, v.7, 65-222

[3] B. Khesin, P. Lee, A nonholonomic Moser theorem and optimal mass transport. arXiv:0802.1551v2 [math.DG], 2008, 31p.

[4] H. J. Sussmann, Orbits of families of vector fields and integrability of distributions. Trans. Amer. Math. Soc., 1973, v.180, 171-188 